\title{
ON JOHN ISAACS' 1952 MEASUREMENTS OF SURFACE-SHIP AND SUBMARINE WAKES
}

\author{
By David Sheres and Walter Munk
}

U PON LEARNING of some very interesting new work on the properties of ship wakes as measured by airborne radar and other modern means $(R$. Gasparovic and D. Johnson, personal communication), we were reminded of some measurements conducted by John Isaacs some 40 years ago and never published (John Dove Isaacs Papers, Scripps Institution of Oceanography-Archives). Isaacs died in June 1980.

In a proposal to the Navy dated 11 June 1952 , Isaacs wrote: "I propose to discuss the possible implications of some conspicuous differences between the hydrodynamic processes involved in the propulsion of surface vessels and snorkeling submarines," and "I propose to examine the velocity and vorticity of the surface wakes at a series of points astern ... by laying out a long flexible cylindrical float (plastic garden hose) and also a series of free floats (mimeograph paper) normal to the wakes, then recording subsequent positions of these floats by aerial photography," and finally, "If these methods give promising results, electronic approaches will be considered."

Figure 1 shows a sketch copied from the proposal. For the surface-ship wake, there is a net momentum in the aft direction; for the snorkeling submarine wake, the momentum integrated across the wake is zero. Figure 2 shows the results of the first sea test conducted on 8 August 1952 two months after the submission of the proposal (Scientific Officers worked quickly during those days), showing the wake signature from the surface vessel U.S.S. EPCER 857 (808 tons with twin screws at $5.7 \mathrm{ft}$ depth). Figure 3 shows the surface

D. Sheres, Center for Marine Science, University of Southern Mississippi, Stennis Space Center, MS 39529, USA. W. Munk, Scripps Institution of Oceanography. University of California, San Diego. La Jolla, CA 92093, USA. wake from the second sea test on 24 October 1952. involving the snorkeling submarine U.S.S. Baya ( 1,500 tons with twin screws at $46.5 \mathrm{ft}$ depth). Also involved were the Scripps Buoy boat (J. Faughan and R. Huffer) and a helicopter with L. Kidd in charge.

Figure 4 shows two sketches copied from Isaacs' field notes. For both surface ship and submarine, the velocity of the hose at the center axis was of order $0.3 \mathrm{ft} / \mathrm{s}$ immediately after passage. But whereas the axial flow is in the expected direction (opposite to the direction of ship travel), it is contrary to the expected direction for the submarine. (This can be seen by the orientation of the bulge of the garden hose relative to the direction of the two vessels.) We could find no specific reference in Isaacs' notes for this peculiar result.

Isaacs measured the positions of the sheets of mimeograph papers on successive photos in an attempt to determine the local flow velocity, vorticity, and convergence. For the surface ship, the flow converges in the areas near the edges of the bulge (this can be seen in Fig. 2) where the hose had significant slack. The regions of convergence were marked by surface slicks.

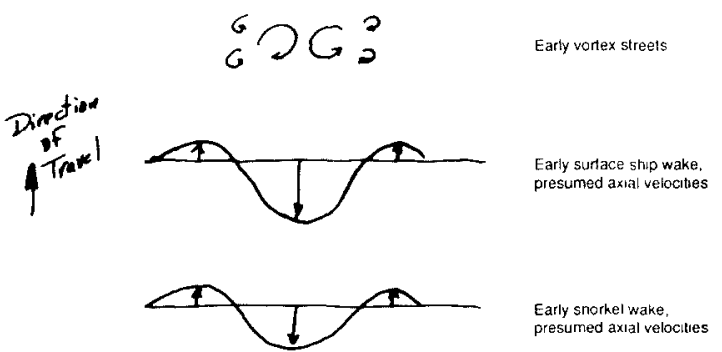

Fig. 1: Expected wake configurations as sketched in proposal. Note the larger axial velocities associated with surface vessel.
“. . . conspicuous

differences between

the hydrodynamic

processes involved in

the propulsion of

surface vessels

and snorkling

submarines" . . . 


\section{EPCER 857}

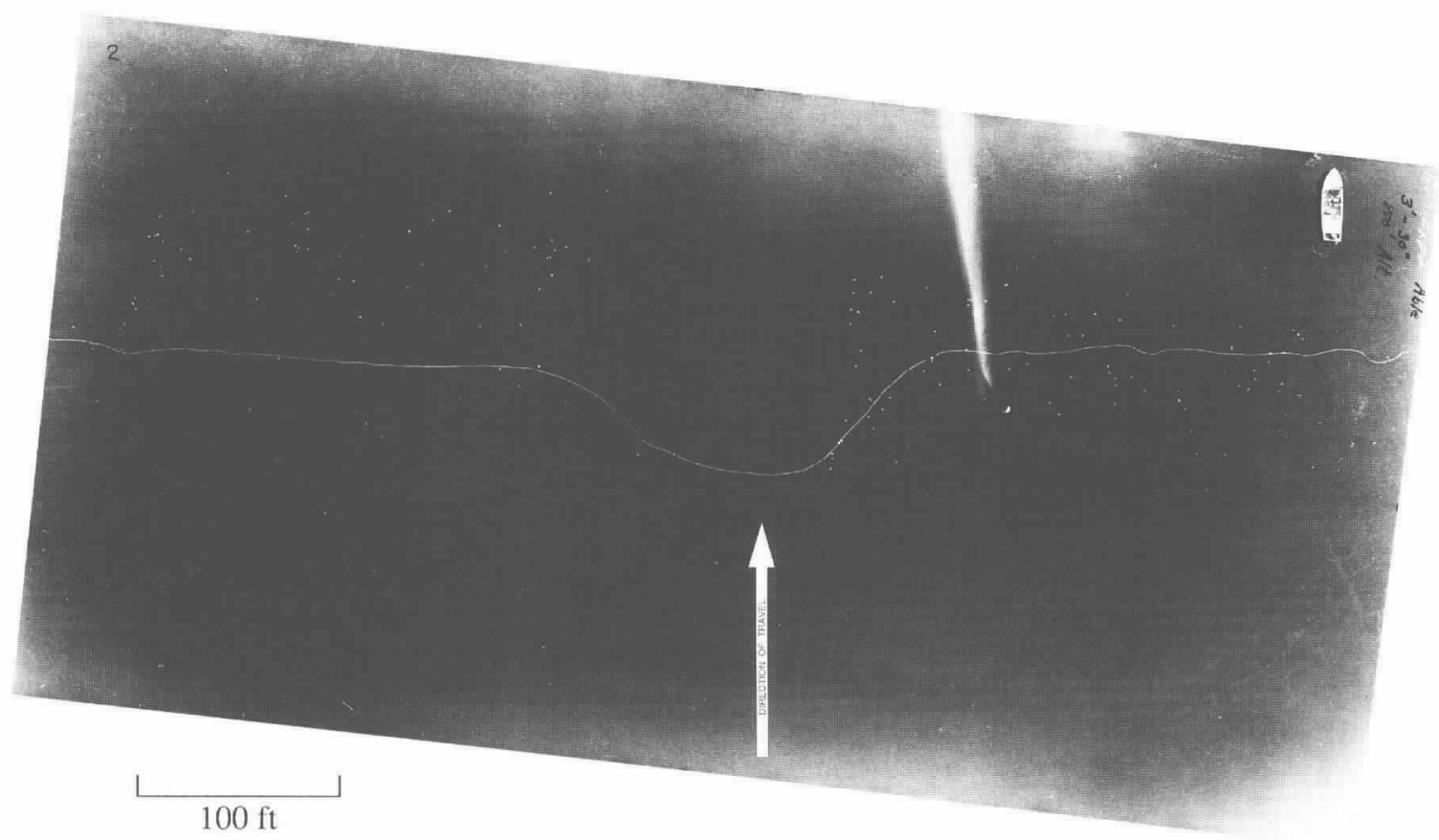

Fig. 2: Picture from a helicopter at $800^{\prime}$ altitude taken $210 \mathrm{~s}$ after passage of surface vessel USS EPCER 857. The garden hose laid across the wake by the buoy boat (in right top corner) appears as a bright line and the sheets of mimeograph paper appear as bright dots.

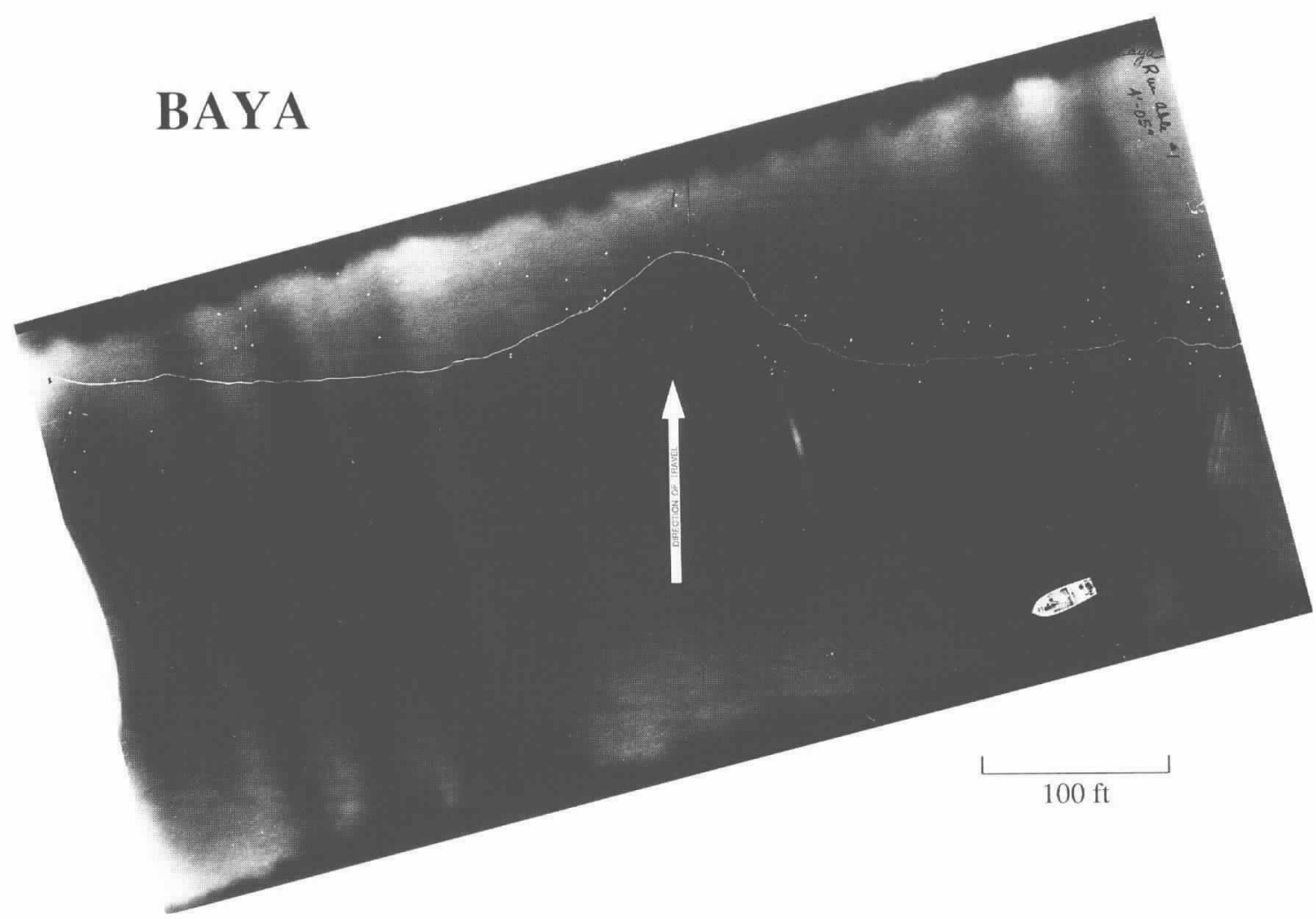

Fig. 3: Picture from a helicopter at 750' altitude taken $245 \mathrm{~s}$ after passage of snorkeling submarine USS $B A Y A$. 


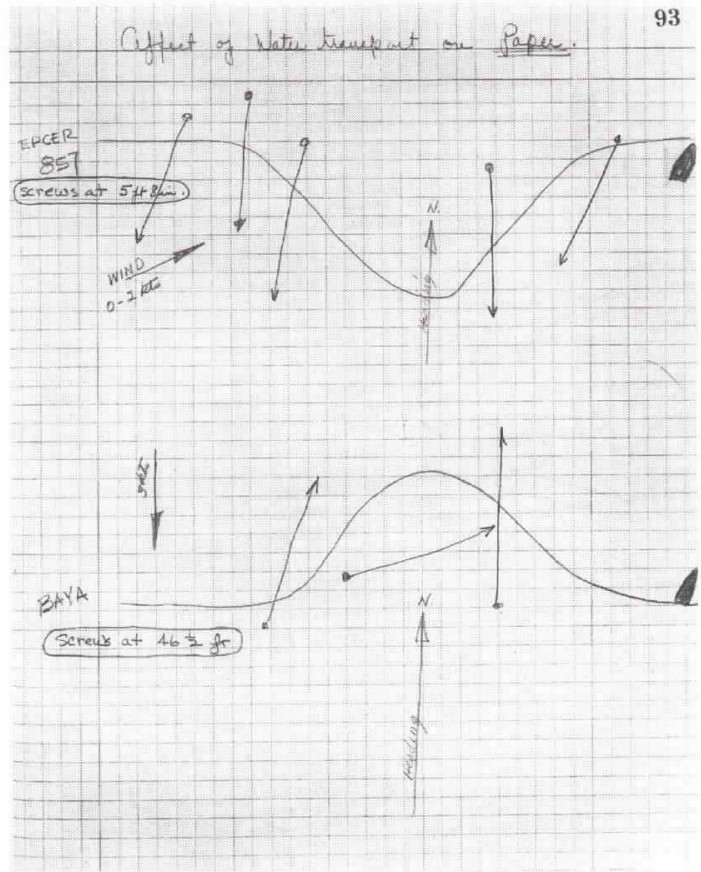

Fig. 4: Isaacs' sketches of observed wake configurations. Arrows give drift direction of mimeograph papers. Solid line shows configuration of garden hose laid across the wake by the Scripps Buoy Boat (sketched at right margin). The direction of drift generated by the submarine is opposite to that sketched in the proposal (Fig. 1).
In the proposal, Isaacs discusses the expected difference between the surface wake by a snorkeling submarine (with almost no wave-making resistance) and the surface wake of a surface vessel: "In the case of any vessel, the thrust of the propellors is equal to the total resistance of the vessel. It follows that for the submarine the total momentum given to the water in the forward axial direction is equal to that in the aft axial direction . . . . the water is not subject to an over-all change in axial momentum by the passage of a submarine at snorkeling depth, and at some infinite distance astern the energy appears principally in vortex and thermal motion." For a surface ship, due to wave and air resistance, "the forward axial momentum given to the water . . . by the effect of total resistance is then only about $60 \%$ of the oppositely directed momentum given by the screws. The wake of a surface ship at some infinite distance astern must therefore display a net velocity aft." This discussion accounts for the difference in the profiles in Fig. 1. It does not give any clue as to why the submarine-induced and surface-ship-induced flows would be in opposite directions.

\section{Acknowledgement}

Scripps Archivist, Deborah Day, has been most helpful in searching for the material.
. . submarine-

induced and surfaceship-induced flows

[were observed] in opposite directions. 